\title{
Diet for diabetic children: a change in emphasis
}

Dietary regulation has always been accepted as a necessary part of the treatment of juvenile onset diabetes mellitus, in order to facilitate the control of blood glucose. In this country it is generally assumed that diabetic control can only be achieved with some form of carbohydrate restriction, and traditionally diets for diabetics have attempted to supply $40 \%$ of calories as carbohydrate. ${ }^{1}$ It is difficult to see how this practice arose because there is little scientific evidence to support it. In Asia and Africa, for economic reasons insulin-requiring diabetics consume diets containing 70 to $80 \%$ carbohydrate with no apparent ill effects. ${ }^{2}$ There is convincing experimental evidence to confirm that diets containing 60 to $85 \%$ of their energy as carbohydrate are well tolerated, and may lead to a reduction in exogenous insulin requirements. $^{34}$ The mechanisms which permit adaptation to a high carbohydrate diet are poorly understood, but probably involve changes in both insulin receptor and post-receptor activity. An increase in the number of insulin receptors ${ }^{5}$ may enhance peripheral sensitivity to insulin. ${ }^{6}$ Increased activity of glycolytic pathway enzymes may also take place. ${ }^{7}$ This adaptation does not occur in the absence of insulin, but if insulin replacement is adequate, carbohydrate handling is restored to a state similar to that in the non-diabetic. ${ }^{3}$

The widespread use of the $10 \mathrm{~g}$ carbohydrate ration, portion or exchange, while having the dogmatic benefit of apparent simplicity, is, as every physiology student knows, a physiological nonsense since all nutrients are capable of conversion directly or indirectly into glucose. The regulation of energy intake is now regarded as having more influence on diabetic control than any individual nutrient, and provided that energy intake does not exceed the needs of growth and expenditure, the proportion of energy contributed by carbohydrate is probably of lesser importance.

A high carbohydrate intake may also have other advantages. The emphasis given to a low carbohydrate intake has resulted in many diabetics eating a diet containing a higher proportion of fat, especially saturated animal fat, than non-diabetics. A higher carbohydrate intake may result in a reduction in the hyperlipidaemia characteristic of the poorly controlled diabetic with consequent improvement in cardiovascular morbidity and mortality.

The higher carbohydrate intakes recommended are not a licence for diabetic children to indulge in sweet shop excesses; a high carbohydrate diet is by no means a 'free' diet. The type of carbohydrate consumed is important too. Sucrose and other simple sugars are excluded from diabetic diets because their rapid absorption produces surges of hyperglycaemia which exogenous insulin cannot prevent. The experimental evidence on which such exclusion was based compared their absorption in aqueous solution with that of starch in food such as bread and potatoes. Isolated preparations of starch however, have a glycaemic effect similar to that of glucose,$^{8}$ and sucrose can have a less acute hyperglycaemic effect than starch. ${ }^{9}$ It seems likely that the earlier studies showed the modifying effect of dietary fibre on postprandial blood glucose levels. It is now known that the digestion of a mixture of carbohydrate and fibre produces less glycaemia than the same amount and type of carbohydrate consumed alone.10 Soluble gel-forming fibres-such as pectin and guar gum - appear to be effective in this respect, but all dietary fibres probably confer some benefit. ${ }^{11}$ An increase in the fibre content of diabetic diets would be rational.

In 1979 the American Diabetes Association ${ }^{12}$ reviewed recent evidence, and issued recommendations concerning the dietary management of diabetics. The Nutrition Subcommittee of the British Diabetes Association has now issued dietary recommendations. Those which are of particular relevance to diabetic children are summarised as follows:

(1) The energy content of the diet in relation to individual energy requirement is probably of the most relevance to dietary control. The proportion of energy derived from carbohydrate need not be regulated at an unduly low level in the effort to normalise glycaemia in the diabetic.

(2) The majority of dietary carbohydrate should be in the form of polysaccharides (for example, bread, potatoes, cereals, beans) and the use of foods which are rich in fibre should be encouraged. Sources of rapidly absorbed mono- and di-saccharides (for example, sweets, chocolates, sweetened drinks) should be excluded except in illness or hypoglycaemic emergency. Refined (fibre-free) starch preparations should be used sparingly.

(3) There may be long-term benefit to the diabetic from a reduction in fat intake, and consequently a more liberal intake of carbohydrate. It is recommended that a maximum of $35 \%$ of dietary energy 
should be obtained from fat and a minimum of $55 \%$ should be supplied by an appropriate carbohydrate. All diets should be nutritionally adequate in vitamins and minerals according to normal physiological requirements.

(4) Fat intake should be reduced primarily at the expense of foods rich in saturated fatty acids such as butter, dairy products such as cheese and cream, and those meat products which have a high content of fat. If part of the remaining fat intake is from polyunsaturated sources, this may improve dietary palatability, and practicality.

(5) In diabetics treated with insulin, the timing of carbohydrate intake remains an important consideration in order to prevent hypoglycaemia. A simple method of estimating and distributing carbohydrate intake is therefore necessary and carbohydrate portions or exchanges are an essential part of dietary management.

(6) Sorbitol, fructose, and diabetic speciality foods which contain these substances are often of little health benefit, since their energy content is equivalent to their conventional counterparts, and the reduction in terms of refined intake is often slight. However, 'dietary' foods which are substantially lower in energy content than their equivalents (for example, low calorie squashes, carbonated drinks, and some tinned fruits), may assist weight loss if required. Saccharine (and any other permitted nonnutritive sweetener) remains an acceptable sugar alternative for diabetics.

Dietary compliance in diabetics is notoriously poor, with no more than $30 \%$ of patients achieving an intake satisfactorily near to that intended.13 The situation in diabetic children is probably worse. How may an effective yet practical diet for diabetic children be best achieved? In childhood dietetics is the art of the possible. ${ }^{14}$ The first requirement is to have the requisite dietetic skills available. The best arrangement will depend on local circumstances. If children are managed in a district general hospital, at least one member of the dietetic staff should have experience not only of diabetes, but of paediatric diabetes as well. In larger paediatric units and children's hospitals, there should be a dietician with specific responsibility for diabetic children. All the members of the diabetic care team-hospital medical and nursing staff, health visitors, family practitioners, and dieticians-should have an agreed common approach so that parents and children are not given conflicting advice.

Many children with diabetes present acutely, and treatment with insulin may be required before the dietician's first interview, and parents may gain the impression that diet is of minor importance. The initial contact is important, and the child's usual eating habits and level of activity should be determined, and used as a basis for dietary prescription. A diet which differs as little as possible from the family's usual routine is much more likely to be followed. Short simple advice is best in the early stages, while the child and his family are coping with injections and urine tests for the first time; elaboration can come later. A home visit by the dietician will allow a more relaxed discussion, and permit an assessment of the family's problems. Imaginative teaching material and methods will engage the attention and co-operation of children and parents much more effectively than diet sheets which list the carbohydrate content of steamed fish or artichokes. We may not approve of convenience foods but many people depend on them. The effectiveness of dietetic teaching should be reviewed at intervals during childhood and especially at adolescence, and the dietician should be seen as an integral member of the follow-up team.

Dietary recommendations for diabetics will no doubt change in future, but at least these recommendations are more firmly based on scientific fact and common sense than the dietetic practices they replace.

\section{References}

1 Truswell A S, Thomas B J, Brown A M. Survey of dietary policy and management in British diabetic clinics. Br Med J 1975; iv: 7-11.

2 Patel J C, Metha A B, Dhirawani M K, Juthani V J, Aiyer L. High carbohydrate diet in the treatment of diabetes mellitus. Diabetologia 1969; 5: 243-7.

3 Brunzell J D, Lerner R L, Porte D, Jr, Bierman E L. Effect of a fat free, high carbohydrate diet on diabetic subjects with fasting hyperglycemia. Diabetes $1974 ; 23$ : 138-42.

4 Simpson R W, Mann J I, Eaton J, Carter R D, Hockaday T D R. High carbohydrate diets and insulin dependent diabetics. $\mathrm{Br}$ Med J 1979; ii: 523-5.

5 Olefsky J M, Reaven G M. Insulin binding in diabetes. Diabetes 1977; 26: 680-8.

${ }^{6}$ Lerner R L, Brunzell J D, Hazzard W R, Porte D, Jr, Bierman E L. Mechanism of improved glucose tolerance on high carbohydrate diets in normals and mild diabetics (abstract). Diabetes 1971; 20: 342-3.

7 Bierman E L, Nelson R. Carbohydrates, diabetes, and blood lipids. World Rev Nutr Diet 1975, 22: 280-7.

8 Wahlqvist M L, Wilmshurst E G, Murton C R, N Richardson $E$ N. The effect of chain length on glucose $\mathrm{\omega}$

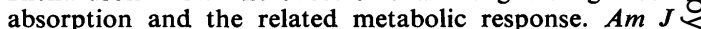
Clin Nutr 1978; 31 : 1998-2001.

9 Thompson R G, Hayford J T, Danney M M. Glucose and insulin responses to diet. Diabetes 1978; 27 : 1020-6. \&

10 Jenkins D J A, Goff D V, Leeds A R, et al. Unabsorbable 7 carbohydrates and diabetes: decreased postprandial hyperglycaemia. Lancet 1976; ii: 172-4.

11 Jenkins D J A, Wolever T M S, Hockaday T D R, et al. Treatment of diabetes with guar gum. Lancet 1977; ii: 779-80. 
12 American Diabetes Association. Principles of nutrition and dietary recommendations for individuals with diabetes mellitus: 1979 . Diabetes $1979 ; 28$ : 1027-30.

13 Thomas B J. How successful are we at persuading diabetics to follow their diet-and why do we sometimes fail? In: Turner $\mathbf{M}$, Thomas $\mathrm{B}$ J, eds. Nutrition and diabetes. London: Libbey, 1981 : 57-66.

${ }^{14}$ Hearnshaw J R. Diet problems specific to diabetic children. In: Turner M, Thomas B J, eds. Nutrition and diabetes. London: Libbey, 1981 : 73-80.

P H W RAYNER Institute of Child Health, The Nuffield Building, Francis Road, Birmingham B16 8ET 